This innovative mass spectrometry probe via polarity-reversal derivatization was confirmed to be a valuable tool for metabolomics study with high sensitivity and separation efficiency in various biological samples.

\section{IDDF2018-ABS-0122 MICRORNA EXPRESSION ANALYSIS OF ADVANCED COLORECTAL CANCER REVEALS A MICRORNA SIGNATURE WITH PROGNOSTIC AND PREDICTIVE VALUE}

Zhaolei Zeng*, Jiahuan Lu, Zhixiang Zuo, Ruihua Xu. Sun Yat-sen University Cancer Center, China

\subsection{6/gutjnl-2018-IDDFabstracts. 14}

Background Prognostic and predictive markers are needed to predict the clinical outcomes of patients with advanced colorectal cancer (CRC) who receive standard first-line treatments. We performed a prospective cohort study in advanced CRC patients to identify a miRNA signature that could predict the benefit of receiving first-line chemotherapy for these patients.

Methods Twenty-one paired tumours and adjacent normal tissues were collected from advanced CRC patients and analysed by miRNA microarrays. Between tumours and normal tissues, 33 miRNAs were differentially expressed and was confirmed by qRT-PCR from another group of 67 patients from a prospective cohort study. A two-miRNA-based signature was obtained using the LASSO Cox regression model based on the association between the expression of each miRNA and the PFS of individual patients. Internal and external validation cohorts, including 40 and 44 patients with advanced CRC, respectively, were performed to prove the prognostic and predictive value of this signature.

Results A signature was built based on two miRNAs, miR125b-2-3 $\mathrm{p}$ and miR-933. CRC patients were classified into low- and high-risk groups for disease progression based on this tool. The patients with low-risk scores generally had better PFS than those with high-risk scores. In the training set, the median PFS in the low- and high-risk groups were 12.00 and 7.40 months, respectively. In the internal validation set, the median PFS in the low- and high-risk groups were 9.90 and 5.10 months, respectively. In the external validation set, the median PFS in the low- and high-risk groups were 9.90 and 6.40 months, respectively. Furthermore, we detected miR125b-2-3 $\mathrm{p}$ associated with CRC cell sensitivity to first-line chemotherapy.

Conclusions Our two-miRNA-based signature was a reliable prognostic and predictive tool for tumour progression in patients with advanced CRC and might be able to predict the benefit of receiving standard first-line chemotherapy in CRC.

\section{IDDF2018-ABS-0125 OVEREXPRESSION OF LNCRNA LINC00460 AFFECTS CELL PROLIFERATION AND APOPTOSIS BY REGULATING KLF2 AND CUL4A EXPRESSION IN COLORECTAL CANCER}

Yifan Lian*, Changsheng Yan, Chuanxing Xiao, Xiang Zhang, Hongzhi Xu, Jianlin Ren. Department of Gastroenterology, Zhongshan Hospital, Xiamen University, Xiamen, Fujian, China

10.1136/gutjnl-2018-IDDFabstracts. 15
Background Emerging evidence has proven that long noncoding RNAs (lncRNAs) play important roles in human colorectal cancer (CRC) biology, while few IncRNAs have been characterised in CRC. Therefore, the functional significance of lncRNAs in the malignant progression of CRC still needs to be further explored.

Methods By utilising publicly available lncRNAs expression profiling data and other publicly available lncRNAs expression profiling data, we screened out LINC00460, whose expression is significantly increased in CRC. The quantitative reverse transcriptase PCR (qRT-PCR) was used to analyse the expression of LINC00460 in 60 CRC tissues and correspond adjacent normal tissues and four CRC cell lines. Gain and loss of function approaches were used to investigate the biological role of LINC00460 both in vitro and in vivo. Bioinformatics analysis followed by qRT-PCR was performed to identify the putative targets of LINC00460, which were further verified by RNA immunoprecipitation (RIP), Chromatin immunoprecipitation (ChIP), Luciferase reporter assays, rescue experiments and western blotting assays.

Results We found a novel lncRNA, LINC00460, whose expression was significantly over-expressed in all three publicly available microarray data. Consistently, qRT-PCR results also verified that LINC00460 was over-expressed in CRC tissues and cells. Furthermore, high LINC00460 expression levels in CRC specimens were correlated with larger tumour size, advanced tumour stage, lymph node metastasis and shorter overall survival. In vitro and in vivo assays of LINC00460 alterations revealed a complexly integrated phenotype affecting cell growth and apoptosis. Mechanistically, LINC00460 repressed kruppel like factor 2 (KLF2) transcription by binding to the enhancer of zeste homolog 2 (EZH2). LINC00460 also functioned as a molecular sponge for miR-149-5 p, antagonising its ability to repress cullin 4A (CUL4A) protein translation.

Conclusions Taken together, our findings support a model in which the LINC00460/EZH2/KLF2 and LINC00460/miR-149$5 \mathrm{p} / \mathrm{CUL} 4 \mathrm{~A}$ crosstalk serve as critical effectors in CRC tumorigenesis and progression, suggesting new therapeutic directions in colorectal cancer.

\section{IDDF2018-ABS-0126 THE PSEUDOGENE DUXAP8 PROMOTES PANCREATIC CANCER CELL PROLIFERATION AND INHIBITES CELL APOPTOSIS BY EPIGENETICALLY SILENCING CDKN1A AND KLF2}

Yifan Lian*, Jiebin Yang, Yikai Lian, Xiaoshen Chen, Xiang Zhang. Department of Gastroenterology, Zhongshan Hospital, Xiamen University, Xiamen, Fujian, China

\subsection{6/gutjnl-2018-IDDFabstracts. 16}

Background Pseudogene has been shown to involve in human cancer biology, but their mechanisms of action are mainly undocumented. Current evidence suggested that pseudogene play a critical role in the regulation of pancreatic cancer cellular processes, such as proliferation, apoptosis, and metastasis. However, only a small proportion of these pseudogene has been functionally characterised.

Methods By utilising publicly available pseudogene expression profiling data and other publicly available pseudogene expression profiling data, we screened out DUXAP8, whose expression is significantly increased in pancreatic cancer (PC) tissues. 